\title{
Hydrotelluration of Alkynes: a Unique Route to Z-Vinyl Organometallics
}

\author{
Maurício L. Vieira ${ }^{a}$, Fabiano K. Zinn ${ }^{b}$ and João V. Comasseto $^{b_{*}}$ \\ a Departamento de Química, Universidade Federal de São Carlos, Via Washington Luiz km 235, CP 676, 13565-905, \\ São Carlos - SP, Brazil \\ ${ }^{\mathrm{b}}$ Instituto de Química, Universidade de São Paulo, Av. Prof. Lineu Prestes 748, CP 26077, 05599-970, \\ São Paulo - SP, Brazil
}

\begin{abstract}
A reação de hidroteluração de alquinos é revisada. A transformação de teluretos vinílicos em organometálicos vinílicos reativos e as reações de acoplamento de teluretos vinílicos com alquinos e organometálicos são apresentadas.
\end{abstract}

\footnotetext{
The hydrotelluration reaction of alkynes is reviewed. The transformation of vinylic tellurides into reactive vinyl organometallics and the coupling reactions of vinylic tellurides with alkynes and organometallics are presented.
}

Keywords: Hydrotelluration, vinylic tellurides, transmetallation, coupling reactions, tellurium.

\section{Introduction}

The hydrometallation of alkynes is the most widely used route to vinylorganometallics, which in turn are valuable in the construction of molecular structures containing carboncarbon double bonds of defined stereochemistry. A number of metal hydrides can participate in this process. However, a few have found use in organic synthesis. The most common hydrometallation reactions involve hydrides of boron ${ }^{1}$, aluminium $^{1 \mathrm{c}, 2}$, zirconium ${ }^{1 \mathrm{c}, 3}$ and $\operatorname{tin}^{1 \mathrm{c}, 4}$. The regio- and stereochemistry of the hydrometallation process depend on the reaction conditions, as well as on the structure of the metal hydride and on the substrate. Usually the reaction displays cis-stereoselectivity giving $E$-organometallics. However isomerization occurs very often leading to mixtures of $Z$ and $E$ vinyl organometallics. The accepted general mechanism for the hydrometallation reaction involves the initial coordination of the alkyne to a vacant orbital of the metal, followed by insertion of the hydrogen-metal bond to a $\pi$ bond of the alkyne (Scheme 1).

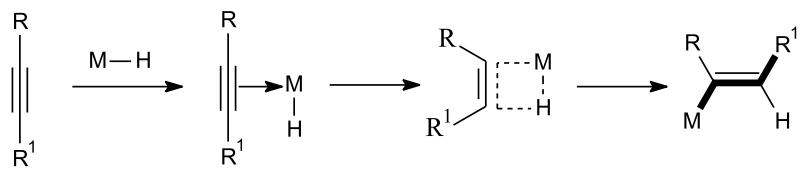

Scheme 1 .

* e-mail: jvcomass@quim.iq.usp.br
The regiochemistry of the hydrometallation depends on the alkyne structure and on the nature of the substituents. The reaction is often performed under transition metal catalysis. Some substituents $(\mathrm{Si}, \mathrm{S}, \mathrm{P})$ direct the metal to the carbon atom linked to them, other $(\mathrm{O}, \mathrm{N})$ direct the metal to the $\beta$ carbon, which is more electron rich and finally, when the alkyne is terminal, mixture of regioisomers can be formed depending on the nature of the $\mathrm{R}$ group (Scheme 2). In this last case, the regioisomer with the metal linked to the less substituted carbon atom of the double bond predominates.

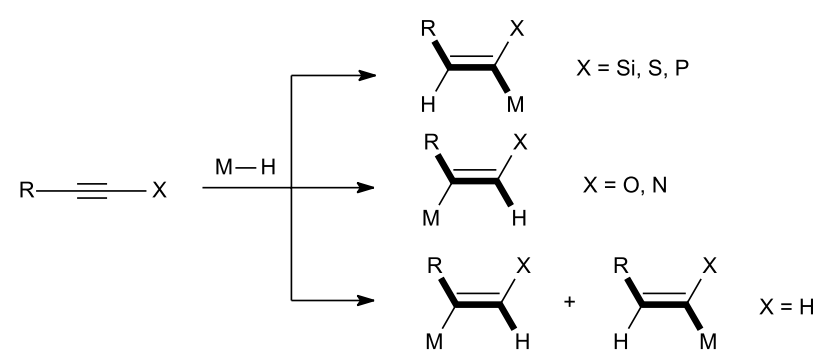

Scheme 2.

In synthetic organic chemistry, the obtained metaloolefins are used for carbon-carbon bond formation exploring the peculiar reactivity of each organometalic, usually making use of palladium catalysts. The most widely used reactions of this type are the Stille $(\mathrm{Sn})^{5}$ and the Suzuki (B) ${ }^{5 b, c, 6}$ couplings. Alternatively, the vinylorganometallics are transformed into reactive intermediates, usually 
vinyllithium and vinylcopper species, which are reacted with a wide range of organic functional groups according to the well established chemistry of organolithium ${ }^{7}$ and organocopper ${ }^{8}$ reagents (Scheme 3 ).

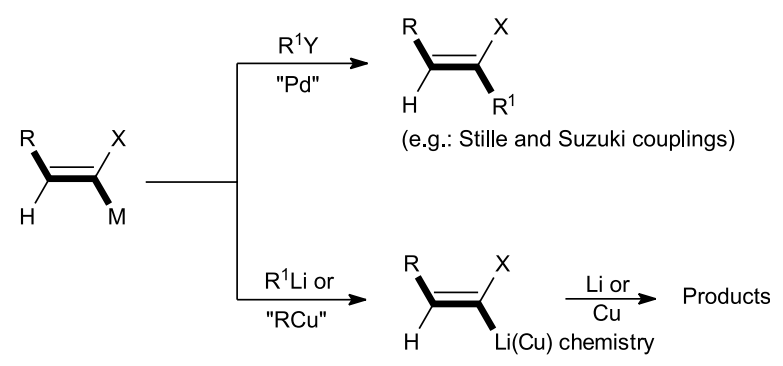

"RCu" = LL ${ }^{1} \mathrm{Cu}(\mathrm{CN}) \mathrm{Li}_{2}\left(\mathrm{~L}, \mathrm{~L}^{1}=\right.$ transferable or non-transferable ligands $)$

Scheme 3.

\section{Hydrotelluration of Alkynes}

As mentioned in the title of this article, the hydrotelluration of alkynes is unique. Contrary to the cis nature of the addition observed for all the other hydrometallation reactions, the hydrotelluration is anti, leading to $Z$-vinylic tellurides, which differently of the vinylorganometallics commented in the introduction, are stereochemically stable. No isomerization of the products of the hydrotelluration of alkynes was reported to date. The hydrotelluration is similar to the hydroselenation ${ }^{9}$ in terms of the steric course, but differs in the stability of the products. Vinylic selenides isomerize easily, giving mixtures of $Z$ and $E$ olefins ${ }^{10}$, what is opposed to the stereochemical stability of the tellurides. Another remarkable difference between hydroselenation and hydrotelluration lays in the stability of selenols and tellurols. Organoselenols, in spite of their evil smell and the high sensitivity to air oxidation, can be isolated and used for preparative purposes. Organotellurols are too unstable to be isolated. Only sterically hindered tellurols can be isolated ${ }^{11}$. In view of the instability of the organotellurols, the hydrotelluration of alkynes is performed using in situ generated organotelurols. The hydrotellurating system is prepared by reacting a diorganoditelluride with sodium borohydride in ethanol ${ }^{12}$. The reduction of the diorganoditelluride can be easily visualized, since the initial solution is dark red. The addition of sodium borohydride to the diorganoditelluride solution leads to the decolorization of the solution with gas evolution. The end of the reduction reaction is indicated by the formation of a colorless solution. The nature of the hydrotellurating agent was never determined and some authors represent it as RTeNa. In view of the protic nature of the solvent, an equilibrium between ionic species present in solution and the solvent can occur, giving rise to a telurol.
However, since the nature of the species present in the ethanolic solution was not yet determined, it seems to be more correct to indicate the hydrotellurating agent as $\left(\mathrm{R}^{2} \mathrm{Te}\right)_{2} / \mathrm{NaBH}_{4}$ (Equation 1).

$$
\mathrm{R} \underset{\mathrm{EtOH}, \mathrm{N}_{2}}{\stackrel{\left(\mathrm{R}^{2} \mathrm{Te}\right)_{2} / \mathrm{NaBH}_{4}}{\longrightarrow}} \stackrel{\mathrm{R}^{1}}{\mathrm{R}^{1}} \mathrm{TeR}_{\mathrm{Te}}^{2}
$$

This is a very practical method to prepare vinylic tellurides in good yields, provided oxygen is excluded from the reaction medium, since oxygen rapidly transforms the hydrotellurating agent into diorganoditelluride, what is indicated by the fast change of the color of the solution from colorless to dark red when air is accidentally introduced in the reaction flask. The only serious drawback of this procedure is when dibutylditelluride is employed as the source of the tellurol, since this reagent is not commercially available and is a very bad smelling compound. A method to generate the hydrotellurating agent which circumvents this problem was recently introduced ${ }^{13}$. This method consists of the insertion of elemental tellurium into a carbon-lithium bond, followed by addition of a proton source such as ethanol or water in stoichiometric amounts. The reaction is performed in deoxygenated tetrahydrofuran under a nitrogen atmosphere. Reaction with the appropriate alkyne gives the vinylic telluride with similar yields to the ones obtained by the ditelluride / sodium borohydride method (Scheme 4).

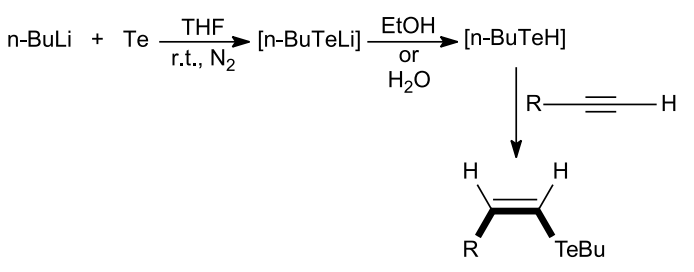

Scheme 4.

An interesting variation of this method consists of its intramolecular version, leading to cyclic tellurides ${ }^{14}$ (Scheme 5).

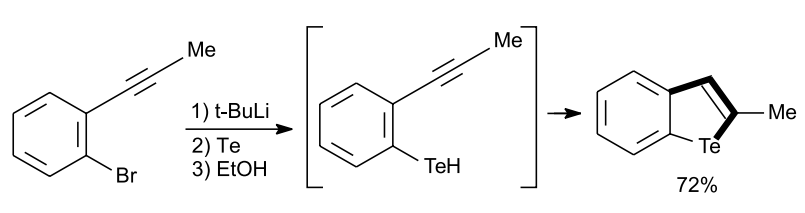

Scheme 5.

For practical reasons, this method will tend to replace the traditional one involving the system diorganoditelluride / sodium borohydride. 
Bis-vinylic tellurides can be prepared by reacting monosubstituted alkynes with a hydrotellurating system prepared from elemental tellurium and sodium borohydride in a mixture of ethanol / aqueous sodium hydroxide ${ }^{15}$. The stereochemistry of the resulting vinylic tellurides is also $Z$ (Equation 2).

$$
2 \mathrm{R} \longrightarrow \mathrm{H} \frac{\mathrm{Te} / \mathrm{NaBH}_{4} / \mathrm{EtOH}, \mathrm{THF}}{\mathrm{NaOH}, \mathrm{H}_{2} \mathrm{O}, \text { reflux, } \mathrm{N}_{2}} \mathrm{R}
$$

Experimentally this reaction is more troublesome than the hydrotelluration using organotellurium species, because a two phase system is formed, that makes it difficult to control the reaction time.

To date a number of vinylic tellurides were prepared by the methods described above. In the following sections we will comment on the influence of the substrate structure on the yields and regiochemistry of the reaction.

\section{Monosubstituted Arylalkynes}

Several vinylic tellurides were prepared starting from monosubstituted arylalkynes. The yields are usually high and the reports in the literature mention that the stereochemistry of the product is $100 \% \mathrm{Z}$. In the same way, only the formation of 1,2-disubstituted vinylic tellurides is reported. The products are almost odorless, crystalline solids or dense pale yellow oils, which can be manipulated for short periods in the presence of the air and indoor light. In addition, these compounds can be recrystalized or chromatographed with no perceptible decomposition. After long standing under refrigeration in the dark, no decomposition or isomerization was observed. However, long exposure to the indoor light leads to the darkening of the products. Another precaution to avoid decomposition is to evaporate the solutions to dryness immediately after the isolation or chromatographic purification, since the compounds in solution are prone to air oxidation. In this way, it is advisable to keep vinylic tellurides protected from light and air. These general comments are valid for the other classes of vinylic tellurides discussed in the forthcoming sections.

In Table 1 are given the structures and yields of the vinylic tellurides prepared from monosubstituted arylalkynes.

\section{Monosubstituted Alkynes Conjugated to a Carbon-Carbon Double Bond}

Alkynes conjugated to double bonds are hydrotellurated in a way similar to arylalkynes (Table 2). The reaction with this class of alkynes is important, since it gives rise to conjugated double bonds of defined stereochemistry. In view of the easy access to enynes of defined stereochemistry $^{22}, Z, Z$ - or $E, Z$-vinylic tellurides can be obtained, as illustrated in Scheme 6.

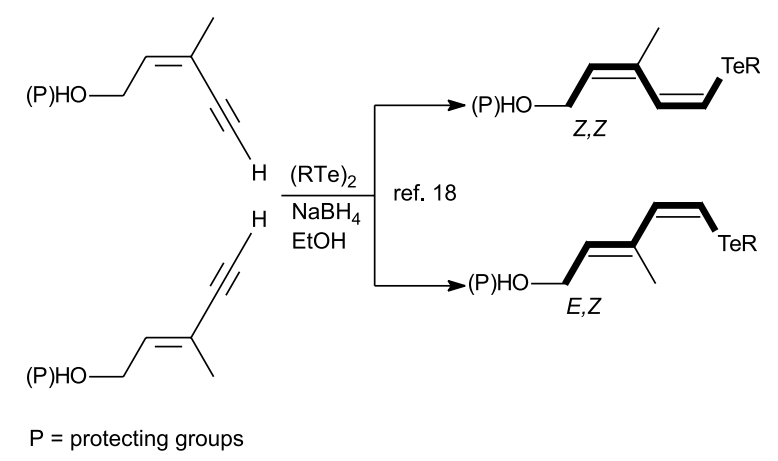

Scheme 6.

In view of the easy transformation of vinylic tellurides into reactive vinylorganometallics (see forthcoming sections), the structures shown in scheme 6 are valuable bifunctional synthons of conjugated olefins (Scheme 7).

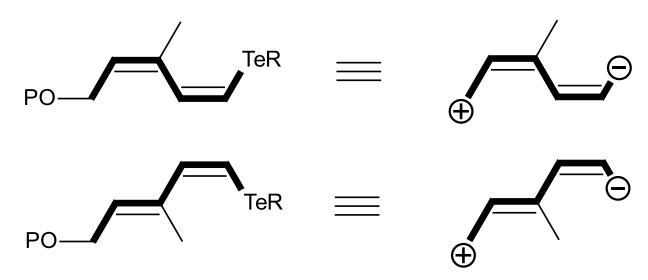

Scheme 7.

As can be observed in Table 2, only one example of hydrotelluration reaction involving a disubstituted alkyne conjugated to a double bond was reported to date (entry 4 , Table 2).

\section{Alkynes Conjugated to Triple Bonds}

Alkynes conjugated to a triple bond are very reactive systems towards hydrotelluration. The reaction occurs in a shorter reaction time when compared to the reactions with the systems commented above. In this case even disubstituted triple bonds give vinylic tellurides in good yields as shown in Table 3. In the case of nonsymmetrical diynes, an order of reactivity was established for the hydrotelluration of triple bonds of the enyne system (terminal > propargylic > alkyl substituted > aryl substituted $)^{25}$. This order of reactivity is reflected in the preferential formation of the vinylic tellurides shown in Scheme 8 . 
Table 1.

\begin{tabular}{|c|c|c|c|c|c|}
\hline Entry $^{\mathrm{a}}$ & $\mathrm{X}$ & $\mathrm{R}$ & Reaction Time $(\mathrm{h})^{\mathrm{b}}$ & Yield (\%) & Ref. \\
\hline $1^{\mathrm{A}}$ & $\mathrm{H}$ & 2-Th & 24 & 60 & 17 \\
\hline $2^{\mathrm{A}}$ & $\mathrm{H}$ & 4-MeOPh & $72^{c}$ & 63 & 18 \\
\hline $3^{\mathrm{A}}$ & $\mathrm{H}$ & $\mathrm{Bu}$ & 1,7 & 93 & 18 \\
\hline $4^{\mathrm{A}}$ & $\mathrm{H}$ & $\mathrm{Bu}$ & 2 & 81 & 17 \\
\hline $5^{\mathrm{A}}$ & $\mathrm{H}$ & $\mathrm{C}\left(\mathrm{CH}_{3}\right)=\mathrm{CH}_{2}$ & 3 & 65 & 15 \\
\hline $6^{\mathrm{A}}$ & $4-\mathrm{Br}$ & $\mathrm{C}\left(\mathrm{CH}_{3}\right)=\mathrm{CH}_{2}^{2}$ & 3 & 77 & 15 \\
\hline $7^{\mathrm{A}}$ & $\mathrm{H}$ & $\mathrm{CH}=\mathrm{CH}_{2}$ & 3 & 76 & 15 \\
\hline $8^{\mathrm{A}}$ & $4-\mathrm{Cl}$ & $\mathrm{CH}=\mathrm{CH}_{2}^{2}$ & 3 & 75 & 15 \\
\hline $9^{\mathrm{A}}$ & $2-\mathrm{COOH}$ & $\mathrm{Me}^{2}$ & 10 & 40 & 19 \\
\hline $10^{\mathrm{A}}$ & 2-COOEt & $\mathrm{Me}$ & 10 & 82 & 19 \\
\hline $11^{\mathrm{A}}$ & 4-Me & $\mathrm{Ph}$ & 20 & 74 & 20 \\
\hline $12^{\mathrm{A}}$ & $4-\mathrm{Cl}$ & $\mathrm{Ph}$ & 20 & 86 & 20 \\
\hline $13^{\mathrm{A}}$ & $4-\mathrm{MeO}$ & $\mathrm{Ph}$ & 20 & 72 & 20 \\
\hline $14^{\mathrm{A}}$ & $2-\mathrm{Cl}$ & $\mathrm{Ph}$ & 20 & 95 & 20 \\
\hline $15^{\mathrm{A}}$ & $3-\mathrm{NO}_{2}$ & $\mathrm{Ph}$ & 20 & 60 & 20 \\
\hline $16^{\mathrm{B}}$ & $\mathrm{H}^{2}$ & Et & $2^{\mathrm{d}}$ & 85 & 21 \\
\hline $17^{\mathrm{B}}$ & $\mathrm{H}$ & $\mathrm{i}-\mathrm{Pr}$ & $2^{\mathrm{d}}$ & 83 & 21 \\
\hline $18^{\mathrm{B}}$ & $\mathrm{H}$ & $\mathrm{Me}$ & $2^{\mathrm{d}}$ & 44 & 21 \\
\hline $19^{\mathrm{D}}$ & $4-\mathrm{Cl}$ & $\mathrm{e}$ & 6 & 98 & 15 \\
\hline $20^{\mathrm{C}}$ & $4-\mathrm{Br}$ & e & 6 & 84 & 15 \\
\hline $21^{\mathrm{C}}$ & 4-Me & $\mathrm{e}$ & 6 & 64 & 15 \\
\hline $22^{\mathrm{C}}$ & $4-\mathrm{MeO}$ & $\mathrm{e}$ & 6 & 65 & 15 \\
\hline $23^{\mathrm{C}}$ & $\mathrm{H}$ & e & 3 & 98 & 15 \\
\hline $24^{\mathrm{C}}$ & $\mathrm{H}$ & e & 36 & 78 & 17 \\
\hline $25^{\mathrm{C}}$ & $4-\mathrm{Cl}$ & $\mathrm{e}$ & 24 & 87 & 17 \\
\hline $26^{\mathrm{C}}$ & 4-Me & e & 36 & 70 & 17 \\
\hline $27^{\mathrm{E}}$ & $\mathrm{H}$ & $\mathrm{Bu}$ & $4^{c}$ & 88 & 13 \\
\hline
\end{tabular}

a) Hydrotelluration system: A) (RTe) $/ \mathrm{NaBH}_{4}$; B) (RTe) $/ \mathrm{KOH} / \mathrm{N}_{2} \mathrm{H}_{4}$; C) Te/KOH; D) Te/NaOH; E) BuTeLi/H $\mathrm{H}^{+}$. All reactions used approximately equimolar amounts of telluride and alkyne. ${ }^{\text {b) }}$ The reactions were carried out in ethanol under reflux. ${ }^{c}{ }^{c} \mathrm{~A}$ THF/ $/ \mathrm{H}_{2} \mathrm{O}$ mixture was used as solvent. ${ }^{\mathrm{d})} \mathrm{A} \mathrm{H}_{2} \mathrm{O} / \mathrm{DMSO}$ mixture was used as solvent. ${ }^{\text {e) }}$ Bis-vinylic tellurides according to eq. 2 were obtained in these cases.

Table 2.

\begin{tabular}{|c|c|c|c|c|c|c|c|c|}
\hline Entry $^{\mathrm{a}}$ & $\mathbf{R}^{1}$ & $\mathbf{R}^{2}$ & $\mathbf{R}^{3}$ & $\mathbf{R}^{4}$ & $\mathbf{R}^{5}$ & Reaction Time $(h)^{c}$ & Yield $(\%)$ & Ref. \\
\hline $1^{\mathrm{A}}$ & $\mathrm{H}$ & $\mathrm{H}$ & $\mathrm{H}$ & $\mathrm{OCH}_{3}$ & $\mathrm{Bu}$ & 28 & 50 & 23 \\
\hline $2^{\mathrm{A}}$ & $\mathrm{H}$ & $\mathrm{CH}_{3}$ & $\mathrm{H}$ & $\mathrm{CH}_{2} \mathrm{OH}$ & $\mathrm{Bu}$ & 2 & 69 & 23 \\
\hline $3^{\mathrm{A}}$ & $\mathrm{H}$ & $\mathrm{H}^{3}$ & $\mathrm{H}$ & $\mathrm{TeBu}$ & $\mathrm{Bu}$ & 2 & 70 & 23 \\
\hline $4^{\mathrm{A}}$ & $\mathrm{CH}_{2} \mathrm{OH}$ & $\mathrm{H}$ & $\mathrm{CH}_{2} \mathrm{OH}$ & $\mathrm{TeBu}$ & $\mathrm{Bu}$ & 0,5 & 83 & 23 \\
\hline $5^{\mathrm{A}}$ & $\stackrel{2}{\mathrm{H}}$ & & $4-\}^{2}$ & $\mathrm{H}$ & $\mathrm{Bu}$ & 12 & 89 & 23 \\
\hline $6^{\mathrm{A}}$ & $\mathrm{H}$ & $\mathrm{CH}_{3}^{\prime}$ & $\mathrm{H}$ & $\mathrm{CH}_{2} \mathrm{OH}$ & $\mathrm{C}_{12} \mathrm{H}_{15}$ & 5 & 80 & 15 \\
\hline $7^{\mathrm{A}}$ & $\mathrm{H}$ & $\mathrm{CH}_{3}^{3}$ & $\mathrm{H}$ & $\mathrm{CH}_{2}^{2} \mathrm{OH}$ & 4-MeOPh & 5 & 70 & 15 \\
\hline $8^{\mathrm{A}}$ & $\mathrm{H}$ & $\mathrm{CH}_{3}^{3}$ & $\mathrm{H}$ & $\mathrm{CH}_{2} \mathrm{OH}$ & 4-MeOPh & 5 & 91 & 15 \\
\hline $9^{\mathrm{A}}$ & $\mathrm{H}$ & $\mathrm{CH}_{3}^{3}$ & $\mathrm{CH}_{2} \mathrm{OH}$ & $\mathrm{H}$ & $\mathrm{C}_{12} \mathrm{H}_{15}$ & 5 & 65 & 15 \\
\hline $10^{\mathrm{A}}$ & $\mathrm{H}$ & $\mathrm{CH}_{3}^{3}$ & $\mathrm{CH}_{2}^{2} \mathrm{OH}$ & $\mathrm{H}$ & $2-\mathrm{MePh}$ & 5 & 75 & 15 \\
\hline $11^{\mathrm{A}}$ & $\mathrm{H}$ & \} & $4-\}^{2}$ & $\mathrm{H}$ & $\mathrm{C}_{12} \mathrm{H}_{15}$ & 5 & 60 & 15 \\
\hline $12^{\mathrm{A}}$ & $\mathrm{H}$ & & $4-\xi$ & $\mathrm{H}$ & 4-MeOPh & 5 & 53 & 15 \\
\hline $13^{\mathrm{A}}$ & $\mathrm{H}$ & $\mathrm{CH}_{3}^{\prime}$ & $\mathrm{CH}_{2} \mathrm{OTHP}$ & $\mathrm{H}$ & $\mathrm{Bu}$ & 4 & 83 & 17 \\
\hline $14^{\mathrm{A}}$ & $\mathrm{H}$ & $\mathrm{H}^{3}$ & $\mathrm{CH}_{2}^{2} \mathrm{OTHP}$ & $\mathrm{H}$ & $\mathrm{Bu}$ & 5 & 89 & 17 \\
\hline $15^{\mathrm{A}}$ & $\mathrm{H}$ & $\mathrm{CH}_{3}$ & ${ }^{2} \mathrm{H}$ & $\mathrm{H}$ & $\mathrm{Bu}$ & 5 & 75 & 17 \\
\hline $16^{\mathrm{B}}$ & $\mathrm{H}$ & $\mathrm{H}^{3}$ & $\mathrm{H}$ & $\mathrm{H}$ & $\mathrm{b}$ & $3^{\mathrm{d}}$ & 3 & 24 \\
\hline $17^{\mathrm{B}}$ & $\mathrm{H}$ & $\mathrm{CH}_{3}$ & $\mathrm{H}$ & $\mathrm{CH}_{2} \mathrm{OH}$ & b & 6 & 77 & 15 \\
\hline $18^{\mathrm{B}}$ & $\mathrm{H}$ & & $4-\{$ & $\mathrm{H}$ & $\mathrm{b}$ & 6 & 6 & 15 \\
\hline $19^{\mathrm{B}}$ & $\mathrm{H}$ & $\mathrm{CH}_{3}$ & $\mathrm{H}$ & $\mathrm{CH}_{2} \mathrm{OTHP}$ & $\mathrm{b}$ & 48 & 60 & 17 \\
\hline $20^{\mathrm{C}}$ & $\mathrm{H}$ & $\mathrm{H}^{3}$ & $\mathrm{CH}_{2} \mathrm{OH}$ & ${ }^{2} \mathrm{H}$ & $\mathrm{Bu}$ & $6^{\mathrm{e}}$ & 77 & 13 \\
\hline
\end{tabular}

a) Hydrotelluration system: A) $\left(\mathrm{R}^{5} \mathrm{Te}\right)_{2} / \mathrm{NaBH}_{4}$; B) Te/KOH; C) BuTeLi/ $\mathrm{H}^{+}$. ${ }^{\text {b) }}$ Bis-vinyllic tellurides according to eq. 2 were obtained in these cases. ${ }^{\mathrm{c}}$ The reactions were performed in ethanol under reflux. ${ }^{\text {d) }}$ Dimethylsulfoxide was used as solvent. ${ }^{\text {e) }}$ A THF/ethanol mixture was used as solvent. 
Table 3.

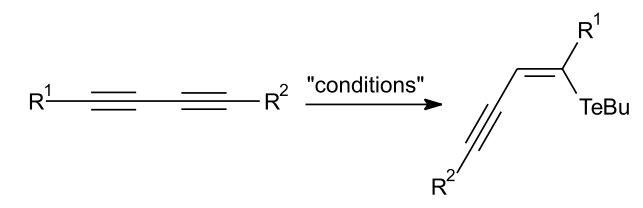

\begin{tabular}{|c|c|c|c|c|c|}
\hline Entry $^{\mathrm{a}}$ & $\mathrm{R}^{1}$ & $\mathrm{R}^{2}$ & Reaction time $(\mathrm{h})^{\mathrm{b}}$ & Yield $(\%)$ & Ref. \\
\hline $1^{\mathrm{A}}$ & $\mathrm{CH}_{2} \mathrm{OH}$ & $\mathrm{CH}_{2} \mathrm{OH}$ & 0.25 & 71 & 23 \\
\hline $2^{\mathrm{A}}$ & $\mathrm{H}$ & $\stackrel{2}{H}$ & 0.25 & 93 & 23 \\
\hline $3^{\mathrm{A}}$ & $\mathrm{CH}_{2} \mathrm{OH}$ & $\mathrm{CH}_{2} \mathrm{OH}$ & 0.25 & 71 & 16 \\
\hline $4^{\mathrm{A}}$ & $\mathrm{H}$ & $\mathrm{H}$ & 0.25 & 93 & 16 \\
\hline $5^{\mathrm{A}}$ & $\mathrm{CH}_{3}$ & $\mathrm{CH}_{3}$ & 2.5 & 81 & 16 \\
\hline $6^{\mathrm{A}}$ & $\mathrm{Ph}$ & $\mathrm{Ph}^{3}$ & 2.5 & 89 & 16 \\
\hline $7^{\mathrm{A}}$ & 4-MePh & 4-MePh & 3 & 73 & 16 \\
\hline $8^{\mathrm{A}}$ & 4-MeOPh & 4-MeOPh & 3 & 68 & 16 \\
\hline $9^{\mathrm{A}}$ & $\mathrm{H}$ & $\mathrm{Ph}$ & 0.16 & 75 & 25 \\
\hline $10^{\mathrm{A}}$ & $\mathrm{H}$ & $\mathrm{C}_{6} \mathrm{H}_{13}$ & 0.28 & 79 & 25 \\
\hline $11^{\mathrm{A}}$ & $\mathrm{CH}_{3}$ & $\mathrm{Ph}$ & 2 & 80 & 25 \\
\hline $12^{\mathrm{A}}$ & $\mathrm{CH}_{2} \mathrm{OH}$ & $\mathrm{Ph}$ & 0.25 & 76 & 25 \\
\hline $13^{\mathrm{A}}$ & $\left(\mathrm{CH}_{2}\right)_{2}^{2} \mathrm{COH}$ & $\mathrm{Ph}$ & 1 & 82 & 25 \\
\hline $14^{\mathrm{A}}$ & $\mathrm{CH}_{2} \mathrm{OH}$ & $\mathrm{C}_{6} \mathrm{H}_{13}$ & 0.58 & 89 & 25 \\
\hline $15^{\mathrm{A}}$ & $\left(\mathrm{CH}_{2}\right)_{2}^{2} \mathrm{COH}$ & $\mathrm{H}$ & 0.16 & 70 & 25 \\
\hline $16^{\mathrm{A}}$ & $\mathrm{H}$ & $\mathrm{H}$ & 3 & 65 & 17 \\
\hline $17^{\mathrm{A}}$ & $\mathrm{Ph}$ & $\mathrm{Ph}$ & 3 & 74 & 17 \\
\hline $18^{\mathrm{B}}$ & $\mathrm{Me}_{3} \mathrm{Si}$ & $\mathrm{Me}_{3} \mathrm{Si}$ & $3^{\mathrm{c}}$ & 37 & 27 \\
\hline $19^{\mathrm{C}}$ & $\mathrm{Ph}$ & $\mathrm{Ph}$ & $8^{\mathrm{d}}$ & 75 & 13 \\
\hline $20^{\mathrm{C}}$ & $\mathrm{CH}_{2} \mathrm{OH}$ & $\mathrm{CH}_{2} \mathrm{OH}$ & $7^{\mathrm{d}}$ & 80 & 13 \\
\hline
\end{tabular}

a) Hydrotelluration system: A) (BuTe) $2 / \mathrm{NaBH}_{4}$; B) Te/NaOH; C) BuTeLi/ $/ \mathrm{H}^{+}$. b) Reaction performed in ethanol under reflux. ${ }^{\mathrm{c})}$ The reaction was performed at room temperature, and tellurophene was obtained. ${ }^{\mathrm{d}} \mathrm{A}$ THF/ethanol mixture was used as solvent.

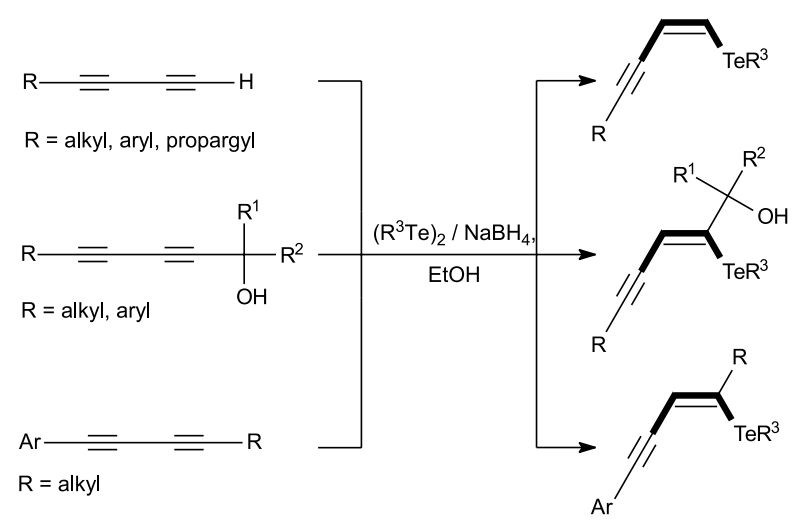

Scheme 8.

\section{Alkynes Bearing Electron Stabilizing Groups}

The hydrotelluration reaction of alkynes bearing keto or ester groups were among the first to be studied ${ }^{12 a}$. Recently, a number of reports dealing with the hydrotelluration of alkynes bearing electron stabilizing groups appeared in the literature (Scheme 9). All systems reported to date give the $Z$ vinylic telluride, with the tellurium moiety linked to the $\beta$-carbon. Several examples of the successful hydrotelluration of internal alkynes of this type were reported (Table 4).

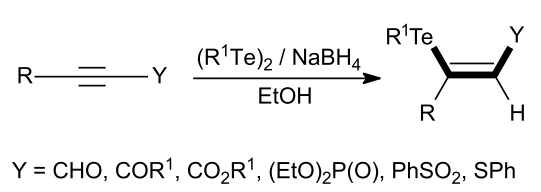

Scheme 9.

\section{Alkylalkynes}

Alkylalkynes are hydrotellurated giving mixture of regioisomers. The Z-1,2-disubstituted vinylic tellurides predominate ${ }^{12 b, c}$. Internal alkynes of this type do not react. Alkylalkynes substituted by an oxygen or nitrogen on carbon 3 also give mixture of regioisomers, but in this case the $Z$ 1,2-disubstituted isomer predominates. On the other hand, secondary or tertiary propargyl alcohols are hydrotellurated to give predominantly Z-1,2-disubstituted vinylic tellurides ${ }^{28}$. Recently, we observed that propargyl alcohol protected by the terc-butyldimethylsilyl group gives almost exclusively the 1,2-disubstituted regioisomer (isomer A) on hydrotelluration $^{37}$ (Scheme 10). The regioisomers can be separated by silica gel column chromatography.

This is the less studied class of alkynes toward hydrotelluration. The limited number of examples studied to date are presented on Table 5. 
Table 4.

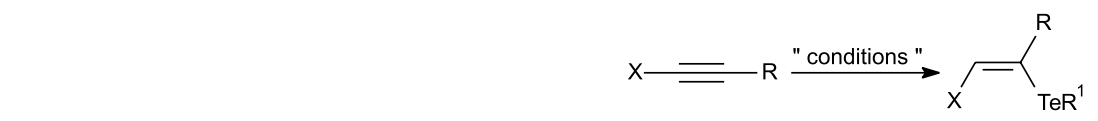

\begin{tabular}{|c|c|c|c|c|c|c|}
\hline Entry $^{a}$ & $\mathrm{R}$ & $\mathrm{R}_{1}$ & $\mathrm{X}$ & Reaction Time (h) & Yield (\%) & Ref. \\
\hline $1^{\mathrm{A}}$ & $\mathrm{H}$ & $\mathrm{Ph}$ & $\mathrm{CO}_{2} \mathrm{Me}$ & 20 & 12 & 28 \\
\hline $2^{\mathrm{A}}$ & $\mathrm{Ph}$ & $\mathrm{Ph}$ & $\mathrm{CO}_{2} \mathrm{Me}$ & 20 & 32 & 28 \\
\hline $3^{\mathrm{B}}$ & $\mathrm{Ph}$ & $4-\mathrm{CH}_{3} \mathrm{COC}_{6} \mathrm{H}_{4}$ & COOEt & 0.5 & 77 & 29 \\
\hline $4^{\mathrm{B}}$ & $\mathrm{Ph}$ & $2,4-\mathrm{MeOPh}$ & COOEt & d & 71 & 30 \\
\hline $5^{\text {в }}$ & $\mathrm{COOH}$ & $2,4-\mathrm{MeOPh}$ & $\mathrm{COPh}$ & 1 & 21 & 30 \\
\hline $6^{\mathrm{B}}$ & $\mathrm{Ph}$ & $2-\mathrm{MePh}$ & COOEt & 0.5 & 58 & 29 \\
\hline $7^{\text {B }}$ & $\mathrm{Ph}$ & 3,5-MeOPh & COOEt & 0.5 & 86 & 31 \\
\hline $8^{\mathrm{B}}$ & $\mathrm{Ph}$ & $3-\mathrm{FPh}$ & COOEt & 0.5 & 60 & 31 \\
\hline $9^{\mathrm{B}}$ & $\mathrm{Ph}$ & 3-MeOPh & COOEt & 0.5 & 98 & 31 \\
\hline $10^{\mathrm{B}}$ & $\mathrm{Ph}$ & 3-MePh & COOEt & 0.5 & 68 & 31 \\
\hline $11^{\mathrm{A}}$ & $\mathrm{Ph}$ & 4-ClPh & $\mathrm{PO}(\mathrm{OEt})$ & Overnight & 63 & 32 \\
\hline $12^{\mathrm{A}}$ & $\mathrm{Ph}$ & 4-FPh & $\mathrm{PO}(\mathrm{OEt})_{2}$ & Overnight & 67 & 32 \\
\hline $13^{\mathrm{A}}$ & $\mathrm{C}_{5} \mathrm{H}_{11}$ & 4-FPh & $\mathrm{PO}(\mathrm{OEt})_{2}$ & Overnight & 59 & 32 \\
\hline $14^{\mathrm{A}}$ & $\mathrm{Ph}$ & $1-\mathrm{C}_{10} \mathrm{H}_{7}$ & $4-\mathrm{MePhSO}_{2}$ & 0.3 & 75 & 32 \\
\hline $15^{\text {B }}$ & $\mathrm{Ph}$ & 4-FPh & $\mathrm{COOMe}^{2}$ & 0.5 & 84 & 29 \\
\hline $16^{\mathrm{B}}$ & $\mathrm{Ph}$ & 4-MeOPh & COOEt & 0.5 & 71 & 29 \\
\hline $17^{\mathrm{B}}$ & $\mathrm{C}_{4} \mathrm{H}_{0}$ & 4-MeOPh & $\mathrm{COPh}$ & d & 68 & 30 \\
\hline $18^{\text {B }}$ & $\mathrm{Ph}$ & 4-MeOPh & $\mathrm{CHO}$ & d & 68 & 30 \\
\hline $19^{\mathrm{A}}$ & $\mathrm{Ph}$ & $4-\mathrm{MePh}$ & $\mathrm{PO}(\mathrm{OEt})$ & Overnight & 59 & 32 \\
\hline $20^{\mathrm{A}}$ & $\mathrm{C}_{5} \mathrm{H}_{11}$ & 4-MePh & $\mathrm{PO}(\mathrm{OEt})_{2}^{2}$ & Overnight & 57 & 32 \\
\hline $21^{\mathrm{B}}$ & ${ }^{5} \mathrm{Ph}^{11}$ & $4-\mathrm{MePh}$ & $\mathrm{COOEt}^{2}$ & 0.5 & 87 & 29 \\
\hline $22^{\mathrm{B}}$ & $\mathrm{Ph}$ & 4-MePh & $\mathrm{CHO}$ & d & 76 & 30 \\
\hline $23^{\mathrm{B}}$ & $\mathrm{H}$ & 4-MePh & COOEt & d & 70 & 30 \\
\hline $24^{\mathrm{B}}$ & $\mathrm{Ph}$ & $4-\mathrm{MePh}$ & $\mathrm{COCH}=\mathrm{C}(\mathrm{TePh})(\mathrm{Ph})$ & 0.3 & 84 & 30 \\
\hline $25^{\mathrm{B}}$ & $\mathrm{Ph}$ & 4-MePh & $\mathrm{COCH}=\mathrm{C}(\mathrm{Ph})\left(\mathrm{NPh}_{2}\right)$ & 0.3 & 68 & 30 \\
\hline $26^{\mathrm{B}}$ & $\mathrm{Ph}$ & $4-\mathrm{MePh}$ & $\mathrm{COCH}=\mathrm{C}(\mathrm{Ph})(\mathrm{OPh})$ & 0.3 & 70 & 30 \\
\hline $27^{\mathrm{B}}$ & $\mathrm{Ph}$ & $\mathrm{Bu}$ & $\mathrm{PO}(\mathrm{OEt})_{2}$ & 4 & 50 & 33 \\
\hline $28^{\mathrm{B}}$ & $\mathrm{Bu}$ & $\mathrm{Bu}$ & $\mathrm{PO}(\mathrm{OEt})_{2}^{2}$ & 5 & 49 & 33 \\
\hline $29^{\mathrm{B}}$ & 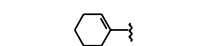 & $\mathrm{Bu}$ & $\mathrm{PO}(\mathrm{OEt})_{2}$ & 4 & 42 & 33 \\
\hline $30^{\text {B }}$ & $\mathrm{H}$ & $\mathrm{Bu}$ & $\mathrm{PO}(\mathrm{OEt})$ & 10 & 85 & 34 \\
\hline $31^{\mathrm{B}}$ & $\mathrm{Ph}$ & $\mathrm{Bu}$ & $\mathrm{PO}(\mathrm{OEt})_{2}$ & 10 & 72 & 34 \\
\hline $32^{\mathrm{B}}$ & $\mathrm{Bu}$ & $\mathrm{Bu}$ & $\mathrm{PO}(\mathrm{OEt})^{2}$ & 10 & 87 & 34 \\
\hline $33^{\mathrm{A}}$ & $\mathrm{H}$ & $\mathrm{Bu}$ & $\mathrm{CHO}$ & $c, d$ & 84 & 35 \\
\hline $34^{\mathrm{B}}$ & $\mathrm{H}$ & $\mathrm{Bu}$ & MorfolCH & $5^{\mathrm{b}}$ & 80 & 15 \\
\hline $35^{\mathrm{B}}$ & $\mathrm{Ph}$ & $\mathrm{Ph}$ & $\mathrm{PO}(\mathrm{OEt})_{2}$ & 4 & 69 & 33 \\
\hline $36^{\mathrm{B}}$ & $\mathrm{Bu}$ & $\mathrm{Ph}$ & $\mathrm{PO}(\mathrm{OEt})_{2}$ & 5 & 60 & 33 \\
\hline $37^{\mathrm{B}}$ & $a$ & $\mathrm{Ph}$ & $\mathrm{PO}(\mathrm{OEt})_{2}$ & 5 & 41 & 33 \\
\hline $38^{\mathrm{B}}$ & $\mathrm{Ph}$ & $\mathrm{Ph}$ & $\mathrm{PhSO}_{2}$ & 10 & 93 & 34 \\
\hline $39^{\mathrm{B}}$ & $\mathrm{Bu}$ & $\mathrm{Ph}$ & $\mathrm{PhSO}_{2}$ & 10 & 94 & 34 \\
\hline $40^{\mathrm{B}}$ & $\mathrm{H}$ & $\mathrm{Ph}$ & $\mathrm{PO}\left(\mathrm{OEt}^{2}\right)_{2}$ & 10 & 92 & 34 \\
\hline $41^{\mathrm{B}}$ & $\mathrm{Ph}$ & $\mathrm{Ph}$ & $\mathrm{PO}(\mathrm{OEt})_{2}$ & 10 & 95 & 34 \\
\hline $42^{\mathrm{B}}$ & $\mathrm{Bu}$ & $\mathrm{Ph}$ & $\mathrm{PO}(\mathrm{OEt})_{2}$ & 10 & 94 & 34 \\
\hline $43^{\mathrm{A}}$ & $\mathrm{Ph}$ & $\mathrm{Ph}$ & $\mathrm{PO}(\mathrm{OEt})_{2}$ & Overnight & 61 & 32 \\
\hline $44^{\mathrm{A}}$ & $\mathrm{Ph}$ & $\mathrm{Ph}$ & $4-\mathrm{MePhSO}_{2}$ & 0.3 & 74 & 32 \\
\hline $45^{\mathrm{A}}$ & $\mathrm{H}$ & $\mathrm{Ph}$ & $4-\mathrm{MePhSO}_{2}$ & 0.3 & 82 & 32 \\
\hline $46^{\mathrm{A}}$ & $\mathrm{C}_{6} \mathrm{H}_{13}$ & $\mathrm{Ph}$ & $4-\mathrm{MePhSO}_{2}^{2}$ & 0.3 & 71 & 32 \\
\hline $47^{\mathrm{C}}$ & $\mathrm{H}^{13}$ & $\mathrm{Ph}$ & $\mathrm{CHO}$ & $\mathrm{c}, \mathrm{e}$ & 80 & 35 \\
\hline $48^{\mathrm{B}}$ & $\mathrm{H}$ & $\mathrm{Ph}$ & COOEt & 0.5 & 95 & 29 \\
\hline $49^{\mathrm{B}}$ & $\mathrm{Ph}$ & $\mathrm{Ph}$ & COOMe & 0.5 & 95 & 29 \\
\hline $50^{\mathrm{B}}$ & $\mathrm{Me}$ & $\mathrm{Ph}$ & COOEt & 0.5 & 83 & 29 \\
\hline $51^{\mathrm{B}}$ & $\mathrm{Ph}$ & $\mathrm{Ph}$ & $\mathrm{COPh}$ & $\mathrm{d}$ & 86 & 30 \\
\hline $52^{\mathrm{B}}$ & $\mathrm{Ph}$ & $\mathrm{Ph}$ & $\mathrm{CHO}$ & d & 88 & 30 \\
\hline $53^{\mathrm{B}}$ & $\mathrm{Ph}$ & $\mathrm{Ph}$ & COOEt & d & 80 & 30 \\
\hline $54^{\mathrm{B}}$ & 1-cyclohexanol & $\mathrm{Ph}$ & $\mathrm{COO} i-\mathrm{Pr}$ & d & 84 & 30 \\
\hline $55^{\mathrm{B}}$ & $\mathrm{Ph}$ & $\mathrm{Ph}$ & $\mathrm{COOH}$ & 1 & 14 & 30 \\
\hline $56^{\mathrm{B}}$ & $\mathrm{Ph}$ & $\mathrm{Ph}$ & $\mathrm{COCH}=\mathrm{C}(\mathrm{TePh})(\mathrm{Ph})$ & $0.3^{\mathrm{b}}$ & 88 & 30 \\
\hline $57^{\mathrm{B}}$ & $\mathrm{H}$ & $\mathrm{Ph}$ & $\mathrm{CO}_{2} \mathrm{Et}$ & 20 & 75 & 28 \\
\hline $58^{\mathrm{B}}$ & $\mathrm{H}$ & $\mathrm{Ph}$ & $\mathrm{CO}_{2}^{2} \mathrm{Et}$ & 20 & 87 & 26,28 \\
\hline $59^{\mathrm{B}}$ & $\mathrm{H}$ & $\mathrm{Ph}$ & $\mathrm{CO}_{2} \mathrm{Me}$ & 20 & 87 & 28 \\
\hline $60^{\mathrm{A}}$ & $\mathrm{Bu}$ & $\mathrm{Ph}$ & $\mathrm{SPh}$ & 3 & 85 & 36 \\
\hline $61^{\mathrm{A}}$ & $\mathrm{Ph}$ & $\mathrm{Ph}$ & $\mathrm{SPh}$ & 3.5 & 70 & 36 \\
\hline $62^{\mathrm{A}}$ & $\mathrm{Bu}$ & $\mathrm{Bu}$ & $\mathrm{SPh}$ & 2 & 81 & 36 \\
\hline $63^{\mathrm{A}}$ & $\mathrm{Ph}$ & $\mathrm{Bu}$ & $\mathrm{SPh}$ & 2.5 & 82 & 36 \\
\hline $64^{\mathrm{A}}$ & $\mathrm{Me}_{3} \mathrm{Si}$ & $\mathrm{Bu}$ & $\mathrm{SPh}$ & 0.75 & 79 & 36 \\
\hline
\end{tabular}

a) Hydrotelluration system was $\left(\mathrm{R}^{1} \mathrm{Te}\right)_{2} / \mathrm{NaBH}_{4}$ at room temperature, and the solvent systems were: A) ethanol; B) ethanol/THF; C) methanol/THF. ${ }^{\text {b) }}$ Reaction carried under reflux. ${ }^{\text {c) }}$ Reaction performed between -5 and $-10{ }^{\circ} \mathrm{C}$. ${ }^{\text {d) }}$ The reaction time was not mentioned. 


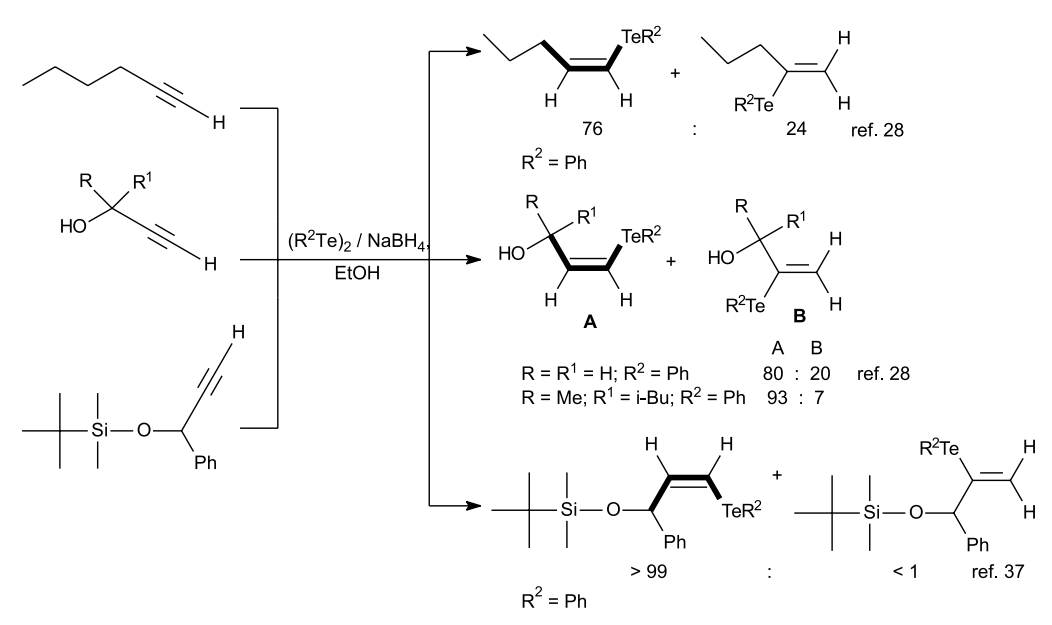

Scheme 10.

Table 5.

\begin{tabular}{|c|c|c|c|c|c|}
\hline Entry ${ }^{\mathrm{a}, \mathrm{d}}$ & $\mathrm{R}$ & $\mathrm{R}^{1}$ & Reaction time $(\mathrm{h})^{\mathrm{c}}$ & Yield $(\%)$ & Ref. \\
\hline $1^{\mathrm{A}}$ & $\mathrm{C}_{6} \mathrm{H}_{13}$ & $\mathrm{Ph}$ & 20 & 25 & 38 \\
\hline $2^{\mathrm{B}}$ & $\mathrm{H}^{13}$ & b & 10 & 94 & 39 \\
\hline $3^{\mathrm{C}}$ & $\mathrm{H}$ & $\mathrm{b}$ & 6 & 54 & 24 \\
\hline $4^{\mathrm{A}}$ & $\mathrm{THPOCH}_{2}$ & $\mathrm{Bu}$ & 5 & 77 & 15 \\
\hline $5^{\mathrm{A}}$ & $\mathrm{C}(\mathrm{OH}) \mathrm{Me}_{2}^{2}$ & $\mathrm{Ph}$ & 5 & 76 & 38 \\
\hline $6^{\mathrm{A}}$ & $\mathrm{CH}_{2} \mathrm{OH}$ & $\mathrm{Ph}$ & 20 & 78 & 28 \\
\hline $7^{\mathrm{A}}$ & $\mathrm{C}\left(\mathrm{Me}_{2}\right)_{2} \mathrm{OH}$ & $\mathrm{Ph}$ & 5 & 76 & 28 \\
\hline $8^{\mathrm{A}}$ & $\mathrm{C}(\mathrm{Me})\left(\mathrm{CH}_{2} \mathrm{CHMe}_{2}\right) \mathrm{OH}$ & $\mathrm{Ph}$ & 20 & 75 & 28 \\
\hline $9^{\mathrm{A}}$ & 1-cyclooctanol & $\mathrm{Ph}$ & 20 & 70 & 28 \\
\hline $10^{\mathrm{D}}$ & $\mathrm{CH}_{2}(\mathrm{Ph})(\mathrm{OTBS})$ & $\mathrm{Bu}$ & 8 & 72 & 13 \\
\hline $11^{\mathrm{E}}$ & $\mathrm{N}-\mathrm{C}$ & b & 6 & 77 & 15 \\
\hline $12^{\mathrm{E}}$ & $\mathrm{N}-\mathrm{CH}_{2}$ & b & 40 & 72 & 17 \\
\hline $13^{\mathrm{F}}$ & $\mathrm{H}$ & $\mathrm{Ph}$ & 5 & 34 & 40 \\
\hline
\end{tabular}

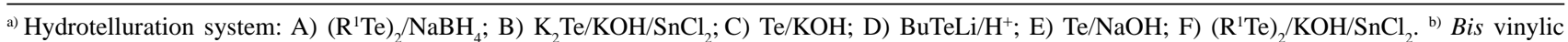
tellurides according to eq. 2 were obtained in these cases. ${ }^{\mathrm{c}}$ The solvents used in the hydrotelluration systems were ethanol (A and $\left.\mathrm{E}\right)$, water (B), water/ HMPA (C), THF/ethanol (D), water/DMSO (F). ${ }^{\text {d) }}$ All reactions were performed under reflux or heating above $100{ }^{\circ} \mathrm{C}$.

\section{Intramolecular Hydrotelluration}

The hydrotellurating methods described in equations 1 and 2 and in Scheme 4 were used to prepare telluriumcontaining heterocycles by intramolecular hydrotelluration of alkynes. Depending on the structure of the starting alkynes, mixtures of endo-dig and exo-dig products (Scheme 11) are formed. In some cases the exclusive formation of one of the possible isomers was observed. The following heterocyclic systems were formed: 3-benzotellurepine $(\mathbf{1})^{14 a}, 1$ benzotellurepine (2) ${ }^{14 \mathrm{~b}-\mathrm{d}}$, tellurochromenes $(\mathbf{3})^{14 \mathrm{~b}}$, benzotellurophenes $\left(\mathbf{4}^{14 \mathrm{e}}, \mathbf{5}^{14 \mathrm{f}}\right)$, tellurophthalides $(\mathbf{6})^{14 \mathrm{~g}}$, tellurochromones $(\mathbf{7})^{14 \mathrm{~h}}$, iso-tellurochromenes $(\mathbf{8})^{14 \mathrm{i}, \mathrm{g}}$, telluroindanes (9) $^{14 i, j}$ and benzotellurazepines (10) ${ }^{141}$ (Scheme 12).

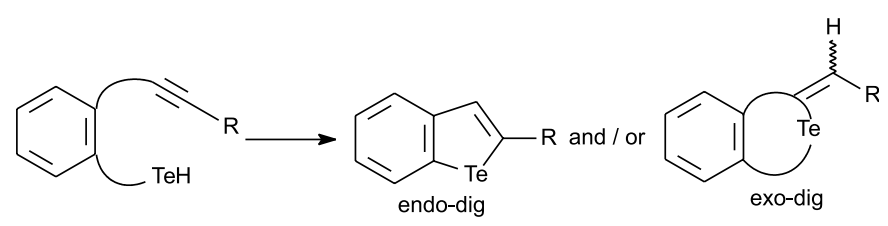


<smiles>C1=Cc2ccccc2C=C[Te]1</smiles>

1<smiles></smiles>

4<smiles>[R]C=C1[Te]C(=O)c2ccccc21</smiles>

6<smiles>[R]C1=CC=Cc2ccccc2[Te]1</smiles><smiles>[R]C=C1C=Cc2ccccc2[Te]1</smiles><smiles></smiles>

5<smiles>[R]c1cc(=O)c2ccccc2o1</smiles><smiles>[R]C1=Cc2ccccc2C[Te]1</smiles>

8<smiles>[R]C=C1[Te]Cc2ccccc21</smiles>

9<smiles>[R]C1=CC(OC)=Nc2ccccc2C1</smiles>

10

Scheme 12.

\section{Tellurium / Metal Exchange Reaction of Z- Vinylic Tellurides}

This is a unique reaction of vinylic tellurides. The other members of the chalcogen family only give the vinylorganometallic on reaction with alkyllithiums if an electronattracting group is present at the $\alpha$ position to the chalcogen. The reaction is very fast and it was reported for the first time in 1982 by Kauffmann ${ }^{41}$ as an extension of the early observation by Seebach ${ }^{42}$ that telluroketal gives 1-lithium-1telluromethane on reaction with organolithiums (Scheme 13).

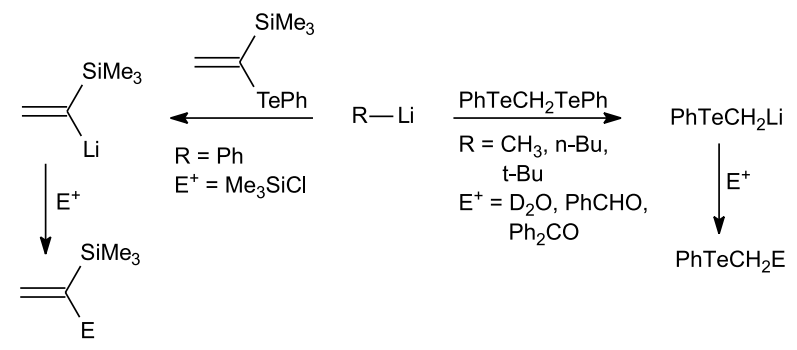

Scheme 13

These results motivated us to explore the hydrotelluration and the Te/Li exchange reactions as synthetic tools.

As no general method for the stereoselective synthesis of vinylic tellurides was available, we undertook a systematic study on the methods to synthesize this class of tellurium compounds, and found that the hydrotelluration of alkynes is the most general and stereoselective method to access

them ${ }^{12 b-e}$. With practical methods to prepare vinylic tellurides in hands, we initiated investigations concerning the Te/Li exchange. It was found that the reaction is very fast and occurs at $-78^{\circ} \mathrm{C}$ with retention of the double bond geometry.

Nowadays the $\mathrm{Te} / \mathrm{Li}$ exchange reaction is well established as a synthetic method and a number of papers were published on this subject ${ }^{12 b-e}$. The tellurium precursors of the $Z$-vinyllithiums are the vinylbutyltellurides, introduced by us as source of Z-vinyllithiums in 1989, since the initially used $Z$-vinylaryltellurides ${ }^{43}$ give mixtures of vinyl and aryllithiums as demonstrated by us in our original report $^{44}$ (Scheme 14).

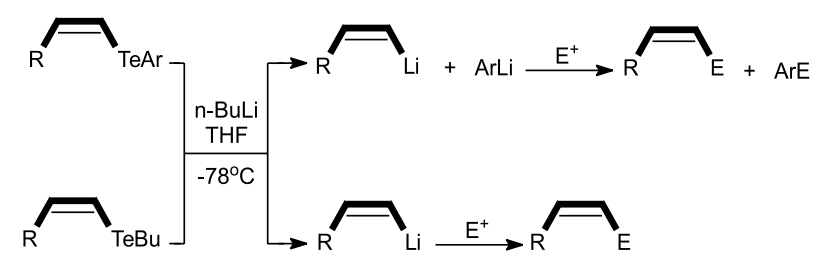

Scheme 14.

This $\mathrm{Te} / \mathrm{Li}$ exchange reaction is noteworthy since it represents the only method available to prepare $Z$ vinyllithiums from alkynes by the sequence hydrometallation/ transmetallation. As commented in the introduction, the other methods give $E$-vinyllithiums. In addition, since lithium is the less electronegative metal among those with synthetic value, this method in principle constitutes a general route to access any $Z$-vinylorganometallic by reaction of the originally formed $Z$-vinyllithium and a salt of a metal more electronegative than lithium (Scheme 15).

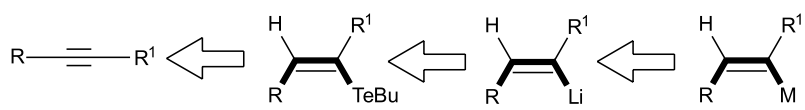

$\mathrm{M}=\mathrm{MgX}, \mathrm{CuCN}, \mathrm{ZnCl}$ and other metals more electronegative than $\mathrm{Li}$

Scheme 15.

Nothwithstanding the practical aspects of this indirect route to Z-vinylorganometallics, many other direct $\mathrm{Te} /$ metal exchange reactions were studied. Scheme 16 summarizes these findings.

$$
\begin{aligned}
& \mathrm{M}=\mathrm{Li}^{15,16,23,25,36,43-45}, \mathrm{Na}^{46} \mathrm{Cal}^{46}, \mathrm{MgX}^{46}, \mathrm{~L}_{2} \mathrm{CuCNL}_{2}{ }^{47}, \mathrm{ZnR}^{48}, \mathrm{ZnCl}^{47 e} \\
& \mathrm{R}^{2}=\text { alkyl, aryl, alkenyl, alkynyl }
\end{aligned}
$$


Among the Te/metal exchange reactions shown in Scheme 14, the direct transformation of vinylic tellurides into "higher order" Z-vinylcyanocuprates is the most synthetically useful in view of the versatility of these intermediates, which deserves comments ${ }^{8}$. The $\mathrm{Te} / \mathrm{Cu}$ exchange reaction was described for the first time by our group and nowadays a number of transformations involving "higher order" Z-vinylcyanocuprates prepared by this method is known ${ }^{12 e, 17,47}$ (Scheme 17).

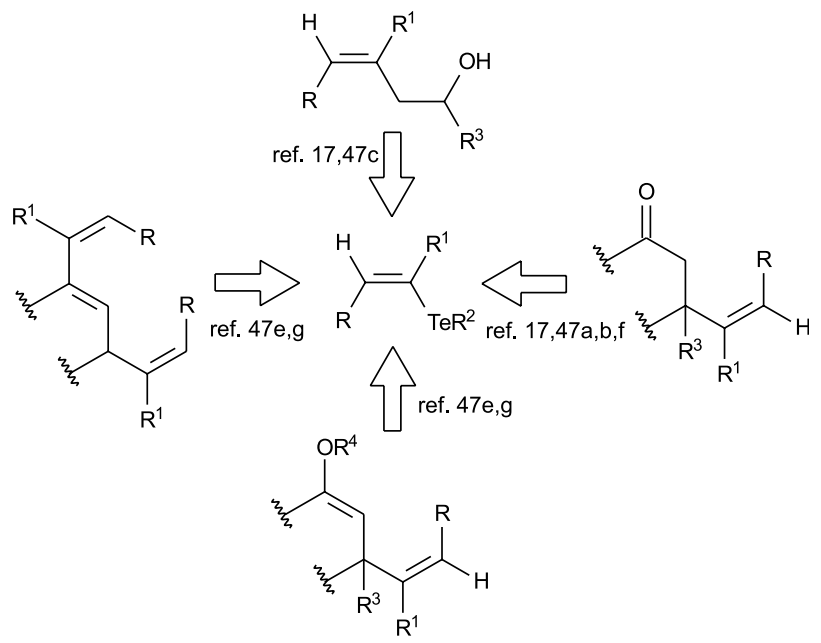

Scheme 17.

\section{Coupling Reactions of Z-Vinylic Tellurides}

Besides the transmetallation reaction of $Z$-vinylic tellurides, the coupling of these intermediates with other organometallics are of synthetic value. The first of such coupling reactions to be developed was the coupling with cuprates. Lower order magnesium or lithium cyanocuprates couple with $Z$-vinylic tellurides in good yield and with retention of the double bond geometry ${ }^{49}$. Higher order magnesium cyanocuprates or mixed lithium-magnesium cyanocuprates react in the same way ${ }^{50}$ (Scheme 18). Similar transformations involving Gilman cuprates ${ }^{51}$ as well as organozinc compounds under palladium catalysis ${ }^{52}$ were also reported.

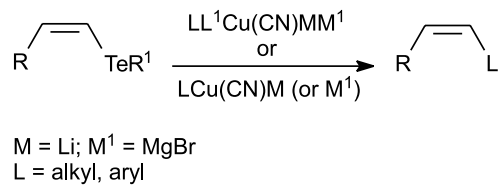

Scheme 18.

Other coupling reaction, which give rise to $Z$-enyne and $Z$-enediyne skeletons involves the transformation of $Z$ - vinylic tellurides into a cuprate, followed by reaction with zinc chloride and then with haloalkynes ${ }^{47 d}$ (Scheme 19).

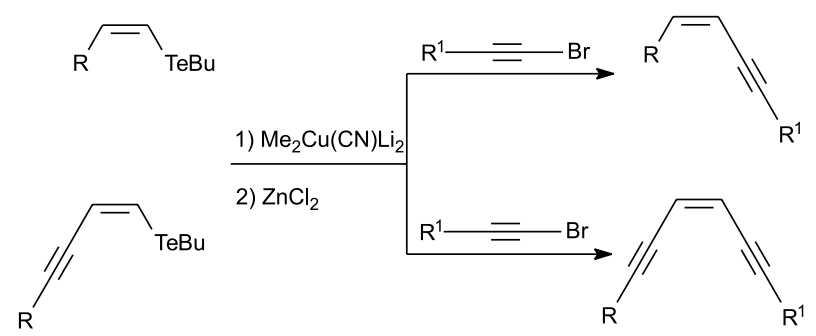

Scheme 19.

The same systems can be obtained by coupling vinylic tellurides with alkynes ${ }^{37,53}$ or zinc acetylides ${ }^{54}$ under palladium catalysis in the presence of an amine (Scheme $20)$.

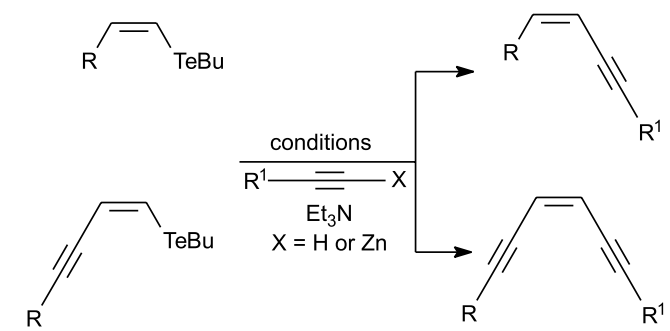

conditions: (a) $\mathrm{PdCl}_{2} / \mathrm{Cul}, \mathrm{MeOH}^{53}$; (b) $\mathrm{Pd}\left(\mathrm{PPh}_{3}\right)_{4} / \mathrm{Cul}$, THF / DMF $(1: 1)^{54}$

Scheme 20.

\section{Conclusion}

The easy and highly stereoselective hydrotelluration of alkynes, coupled to the transformation of the resulting $Z$-vinylic tellurides into reactive metallic species make these reactions a valuable methodology for assembling highly unsaturated systems present in a number of natural products $^{55}$. The synthesis of some biologically active compounds with this structural feature are underway in our group.

Acknowledgements The authors thank FAPESP and CNPq for support.

\section{References}

1. (a) Budnov, Y. N.; Bogdanov, V. S.; Mikhailov, B. M. Izv. Akad. Nuak SSSR, Ser. Khim. 1970, 2416; (b)

Brown, H. C. Organic Synthesis via Boranes, John Wiley, New York, 1975; (c) Bateson, J. H.; Mitchell, M. B. (Ed) Organometallic Reagents in Organic Synthesis, Academic Press, London, 1994. 
2. Eisch, J. J.; Foxton, M. W. J. Org. Chem. 1971, 36, 3520 .

3. Vicent, P.; Beaucourt, J. P.; Picht, L. Tetrahedron Lett. 1982, 23, 63.

4. Leusink, A. J.; Budding, H. A.; Marsman, J. W. J. Organometal. Chem. 1967, 9, 286.

5. (a) Labadie, J. W.; Tueting, D.; Stille, J. K. J. Org. Chem. 1983, 48, 4634; (b) Beller, M.; Bolm, C. (Ed.) Transition Metals for Organic Synthesis, Wiley-VCH, Weinheim, 1998; (c) Franzén, R. Can. J. Chem. 2000, $78,957$.

6. Miyaura, N.; Yanagi, T.; Suzuki, A. Synth. Comun. 1981, 11, 513.

7. Snieckus, V.; Gray, M.; Tinkl, M. Lithium In Comprehensive Organometallic Chemistry, vol. 11, Abel, E. W.; Stoke, F. G. A.; Wilkinson, G. (Eds), Pergamon, Oxford, 1995.

8. (a) Lipshutz, B. H.; Sengupta, S. Org. React. 1992, 41, 135; (b) Taylor, R. J. K. (Ed.) Organocopper Reagents, Oxford University Press, Oxford, 1994.

9. Comasseto, J. V.; Ferreira, J. T. B.; Petragnani, N. J. Organomet. Chem. 1981, 216, 287.

10. Fryzuk, M. D.; Bates, G. S.; Stone, C. J. Org. Chem. 1991, 56, 7201.

11. Dabbousi, B. O.; Bonasia, P. J.; Arnold, J. J. Am. Chem. Soc. 1991, 113, 3186.

12. (a) Buzilova, S. R.; Vereshchagin, L. I.; Sadekov, I. D.; Minkin, V. I. J. Org. Chem. (USSR) 1976, 46, 932; For reviews see: (b) Comasseto, J. V. Rev. Heteroatom. Chem. 1993, 9, 61; (c) Petragnani, N. Tellurium Reagents in Organic Synthesis, Academic Press, London, 1994; (d) Comasseto, J. V.; Lo, W. L.; Petragnani, N.; Stefani, H. A. Synthesis 1997, 373; (e) Comasseto, J. V.; Barrientos-Astigarraga, R. E. Aldrichimica Acta 2000, 33, 66.

13. Zeni, G.; Formiga, H. B.; Comasseto, J. V. Tetrahedron Lett. 2000, 41, 1311.

14. (a) Sashida, H.; Kurahashi, H.; Tsuchiya, T. J. Chem. Soc., Chem. Commun. 1991, 802; (b) Sashida, H.; Ito, K.; Tsuchiya, T. J. Chem. Soc., Chem. Commun. 1993, 1493; (c) Sashida, H.; Ito, K.; Tsuchiya, T. Chem. Pharm. Bull. 1995, 43, 19; (d) Sashida, H. Heterocycles 2000, 52, 49; (e) Sashida, H.; Sadamori, K.; Tsuchiya, T. Synth. Commun. 1998, 28, 713; (f) Sashida, H.; Yasuike, S. J. Heterocycl. Chem. 1998, 35, 725; (g) Sashida, H.; Kawamukai, A. J. Heterocycl. Chem. 1998, 35, 165; (h) Sashida, H. Synthesis 1998, 745; (i) Sashida, H.; Ohyanagi, K. J. Chem. Soc., Perkin Trans 1 1998, 2123; (j) Sashida, H. Synthesis 1999, 1866; (1) Sashida, H. Heterocycles 1998, 48, 631; For a review see: Sashida, H. Rev. Heteroatom. Chem. 2000, 22, 59.
15. Barros, S. M.; Dabdoub, M. J.; Dabdoub, V. M.; Comasseto, J. V. Organometallics 1989, 8, 1661.

16. Dabdoub, M. J.; Dabdoub, V. B.; Comasseto, J. V. Tetrahedron Lett. 1992, 33, 2261.

17. Tucci, F. C.; Chieffi, A.; Comasseto, J. V.; Marino, J. P. J. Org. Chem. 1996, 61, 4975.

18. Dabdoub, M. J.; Dabdoub, V. B.; Comasseto, J. V.; Petragnani, N. J. Organomet. Chem. 1986, 308, 211.

19. Luxen, A.; Christiaens, L. Renson, M. J. Org. Chem. 1980, 45, 3535 .

20. Ohe, K.; Takahashi, H.; Uemura, S.; Sugita, N. J. Org. Chem. 1987, 52, 4859.

21. Potapov, V. A.; Amosova, S. V. Phosphorous, Sulfur and Silicon 1993, 79, 277.

22. Brandsma, L. Preparative Acetylenic Chemistry, Elsevier Science Publishers B. V., New York, 1988.

23. Dabdoub, M. J.; Dabdoub, V. B.; Guerrero Jr., P. G.; Silveira, C. C. Tetrahedron 1997, 53, 4199.

24. Trofimov, B. A.; Amosova, S. V.; Gusarova, N., K.; Musorin, G. K. Tetrahedron 1982, 38, 713.

25. Dabdoub, M. J.; Dabdoub, V. B. Tetrahedron 1995, $51,9839$.

26. (a) Uemura, S.; Fukuzawa, S. Tetrahedron Lett. 1982, 23, 1181; (b) Ohe, K.; Takahashi, H.; Uemura, S.; Sugita, N. Nippon Kagaku Kaishi 1987, 1469.

27. Barton, T. J.; Roth, R. W. J. Organometal. Chem. 1972 , 39, C66.

28. Takahashi, H.; Ohe, K.; Uemura, S. Nippon Kagaku Kaishi 1987, 1508.

29. Detty, M. R.; Murray, B. J.; Smith, D. L.; Zumbulyadis, N. J. Am. Chem. Soc. 1983, 105, 875.

30. Buzilova, S. R.; Sadekov, I. D.; Lipovich, T. V. Filippova, T. M.; Vereshchagin, L. I. J. Gen. Chem. (USSR) 1977, 47, 1828.

31. Detty, M. R.; Murray, B. J. J. Am. Chem. Soc. 1983, $105,883$.

32. Huang, X.; Liang, C. -G.; Xu, Q.; He, Q. -W. J. Org. Chem. 2001, 66, 74.

33. Braga, A. L.; Alves, E. F.; Silveira, C. C.; Andrade, L. H. Tetrahedron Lett. 2000, 41, 161.

34. Jang, W. B.; Oh, D. Y.; Lee, C. -W. Tetrahedron Lett. 2000, 41, 5130 .

35. Mo, X. -S.; Huang, Y. -Z. Tetrahedron Lett. 1995, $36,3539$.

36. Dabdoub, M. J.; Dabdoub, V. B.; Pereira, M. A. Tetrahedron Lett. 2000, 42, 1595.

37. Barrientos-Astigarraga, R. E.; Castelani, P.; Comasseto, J. V.; Formiga, H. B.; Silva, N. C.; Sumida, C. Y.; Vieira, M. L. J. Organomet. Chem. 2001, 623, 43.

38. Ohe, K.; Takahashi, H.; Uemura, S.; Sugita, N. J. Organomet. Chem. 1987, 326, 35. 
39. Trofimov, B. A.; Tatarinova, A. A.; Gusarova, N. K.; Amosova, S. V.; Sinegovskaya, L. M.; Bzhezovskii, V. M. J. Org. Chem. (USSR) 1983, 2181.

40. Potapov, V. A.; Kashik, A. S.; Gusarova, N. K.; Minkin, V. I.; Sadekov, I. D.; Trofimov, B. A.; Amosova, S. V. J. Org. Chem. (USSR) 1987, 23, 596.

41. (a) Kauffmann, T. Angew. Chem. Int. Ed. Engl. 1982, 21, 410; (b) Kauffmann, T.; Allers, H. Chem. Ber. 1983, 116, 1001.

42. Seebach, D.; Beck, A. K. Chem. Ber. 1975, 108, 314.

43. Hiiro, T.; Kambe, N.; Ogawa, A.; Miyoshi, N.; Murai, S.; Sonoda, N. Angew. Chem. Int. Ed. Engl. 1987, 26, 1187.

44. Barros, S. M.; Comasseto, J. V.; Berriel, J. Tetrahedron Lett. 1989, 30, 7353.

45. (a) Ogawa, A.; Tsuboi, Y.; Obayashi, R.; Yokoyama, K.; Ryu, I.; Sonoda, N. J. Org. Chem. 1994, 59, 1600; (b) Dabdoub, M. J.; Begnini, M. L.; Cassol, T. M.; Guerrero Jr., P. G.; Silveira, C. C. Tetrahedron Lett. 1995, 36, 7623; (c) Dabdoub, M. J.; Dabdoub, V. B.; Pereira, M. A.; Zukerman-Schpector, J. J. Org., Chem. 1996, 61, 9503; (d) Huang, Y. -Z.; Mo, X. -S. Tetrahedron Lett. 1998, 39, 1945; (e) Dabdoub, M. J.; Jacob, R. G.; Ferreira, J. T. B.; Dabdoub, V. B.; Marques, F. Tetrahedron Lett. 1999, 40, 7159.

46. Kanda, T.; Sugino, T.; Kambe, N.; Sonoda, N. Phosphorous, Sulfur, Silicon Relat. Elem. 1992, 67, 103.
47. (a) Comasseto, J. V.; Berriel, J. N. Synth. Commun. 1990, 20, 1681; (b) Tucci, F. C.; Chieffi, A.; Comasseto, J. V. Tetrahedron Lett. 1992, 33, 5721; (c) Marino, J. P.; Tucci, F. C.; Comasseto, J. V. Synlett 1993, 761; (d) Araujo, M. A.; Comasseto, J. V. Synlett 1995, 1145; (e) Barrientos-Astigarraga, R. E.; Moraes, D. N.; Comasseto, J. V. Tetrahedron Lett. 1999, 40, 265; (f) Araujo, M. A.; Ellensohn, R. M.; BarrientosAstigarraga, R. E.; Comasseto, J. V. Tetrahedron Lett. 1999, 40, 5115; (g) Moraes, D. N.; BarrientosAstigarraga, R. E.; Castelani, P.; Comasseto, J. V. Tetrahedron 2000, 56, 3327.

48. Terao, J.; Kambe, N.; Sonoda, N. Tetrahedron Lett. 1996, 37, 4741.

49. Chieffi, A.; Comasseto, J. V. Synlett 1995, 671.

50. Chieffi, A.; Comasseto, J. V. Tetrahedron Lett. 1994, $35,4063$.

51. Ogawa, A.; Tsuboi, Y.; Obayashi, R.; Yokoama, K.; Ryu, L.; Sonoda, N. J. Org. Chem. 1994, 59, 1600.

52. Dabdoub, M. J.; Dabdoub, V. B.; Marino, J. P. Tetrahedron Lett. 2000, 41, 433.

53. Zeni, G.; Comasseto, J. V. Tetrahedron Lett. 1999, 40, 4619.

54. Dabdoub, M. J.; Dabdoub, V. B.; Marino, J. P. Tetrahedron Lett. 2000, 41, 437.

55. Faulkner, D. J. Nat. Prod. Rep. 1998, 15, 113.

Received: June 25, 2001

Published on the web: August 15, 2001

FAPESP helped in meeting the publication costs of this article. 